\title{
An Evaluation of Esophageal Temperature Management in Cases Longer than 72h
}

M. Naiman, PhD'; A. Markota, MD²; J. Haymore, DNP; N. Badjatia, MD³; A. Hegazy, MBBCh FRCPC ${ }^{4}$; E. Kulstad, MD, MS ${ }^{5}$

1) University of Illinois at Chicago, 2) University Medical Centre Maribor, 3) University of Maryland, 4) University of Western Ontario, 5) University of Texas Southwestern

\section{Background}

Targeted temperature management (TTM) is an important aspect of post-resuscitation care. ${ }^{1,2}$

Providing therapeutic hypothermia ${ }^{3}$ and avoiding post-cooling hyperthermia ${ }^{4}$ are both important to patient outcomes. While clinical evidence does not indicate that any method is superior ${ }^{5}$, the selected modality must steadily maintain target temperature throughout treatment to be effective.

\section{Purpose}

Esophageal Temperature Management (ETM, Figure 1) is a new TTM modality. Originally the device was ap proved for $36 \mathrm{~h}$ treatment durations, but this was ex tended to $120 \mathrm{~h}$ in 2016 . The purpose of this study is to evaluate device performance and user experience during extended duration cases.

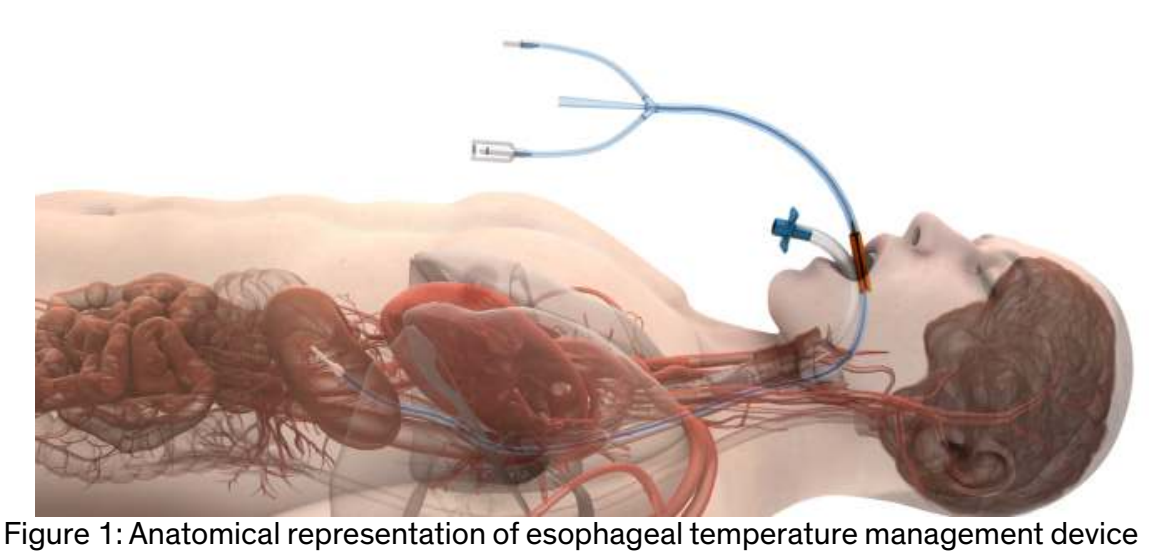

\section{Methods}

Retrospective analysis of patients treated with ETM for at least $72 \mathrm{~h}$ with core temperature recorded at least every 4h. Data points were pooled to calculate descriptive statistics and plotted on a control chart to visualize the proportion of readings that remained within $\pm 1^{\circ} \mathrm{C}$ of target. User experience was documented through a short phone interview with providers who placed the device.

\section{References}

1. Bernard SA, Gray TW, Buist MD, et al. N Engl J Med. 2002;346(8):557-563. 2. The HACA. N Engl J Med. 2002;346(8):549-556

3. Nielsen N, Wetterslev J, Cronberg T, et al. N Engl J Med. 2013;369(23):2197-2206 4. Bro-Jeppesen J, Hassager C, Wanscher M, et al. Resuscitation. 2013;84(12):1734-1740. 5. Deye N, Cariou A, Girardie P, et al. Circulation. 2015;132(3):182.

\section{Results}

18 records were included, providing 1237 data points for analysis. Indications for temperature management in this cohort included treatment with hypothermia post cardiac arrest and refractory fever control. The average treatment duration was $137.7 \mathrm{~h}$ (range $72 \mathrm{~h}-452 \mathrm{~h}$ ) and the target temperature ranged from $33-38^{\circ} \mathrm{C} .1030$ measures $(83.3 \%)$ were within $\pm 1^{\circ} \mathrm{C}$ of target during the protocol.

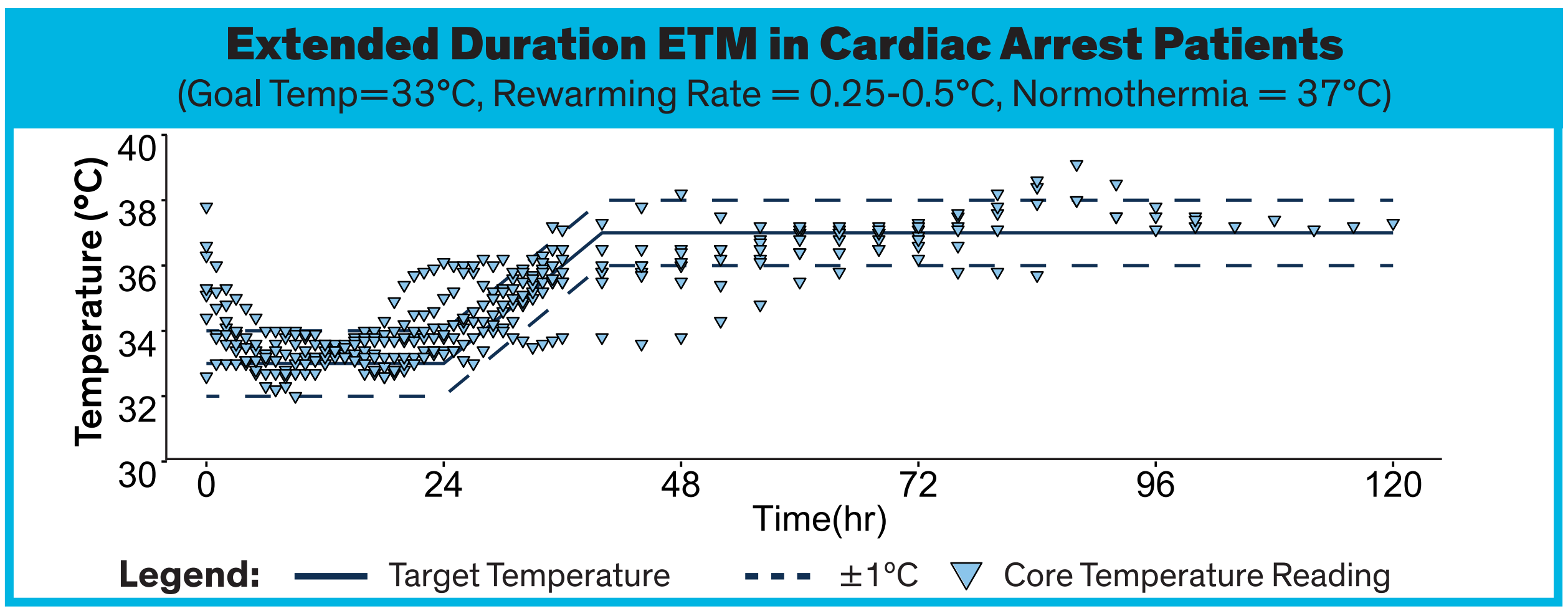

Extended Duration ETM in Refractory Fever Patients

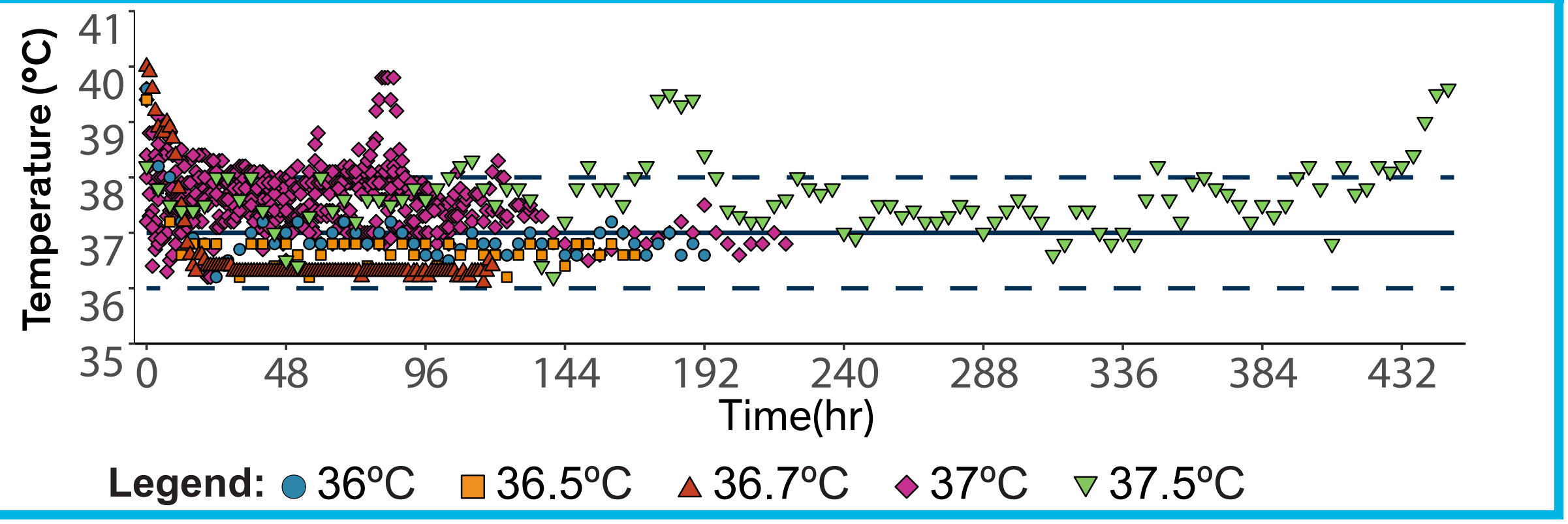

\section{Conclusion}

This initial analysis suggests that esophageal heat transfer meets the needs of extended duration target temperature management protocols. Additional research is needed to confirm anecdotal reports conveyed during user interviews, such as reduced shivering burden and reduced sedation.
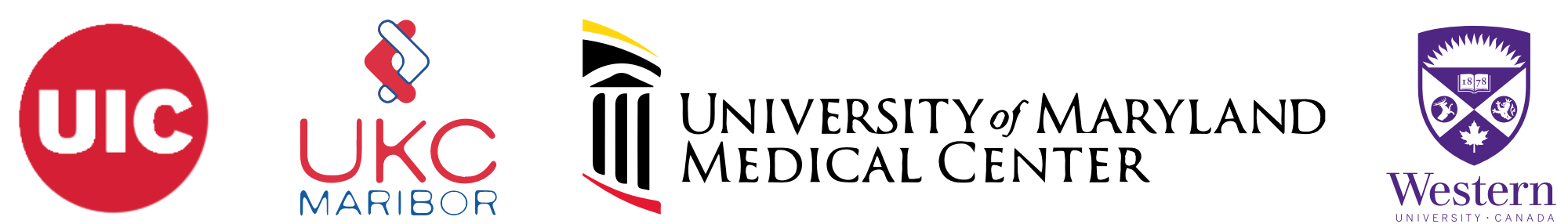

UTSouthwestern Medical Center

Disclosures: Drs. Naiman and Kulstad have a financial relationship with Attune Medical, the manufacturer of the devices used in this evaluation. Drs. Badjatia, Haymore, Hegazy, and Markota received some of the devices used in the evaluation free of charge. 